\title{
Cardiac Arrhythmia Due to Intraorbital Hematoma During Oculoplastic Surgery Under Local Anesthesia: A Case Report
}

\author{
Catia Sousa Govêia ${ }^{1}$, Denismar Borges de Miranda ${ }^{2 *}$, Larissa Govêia Moreira ${ }^{3}$ and Luís Cláudio \\ Araújo Ladeira $^{1}$ \\ ${ }^{1}$ Department of Anesthesiology, Universidade de Brasília, Brasília, Distrito Federal, Brazil \\ ${ }^{2}$ Municipal Health Department of Goiânia, Goiás, Brazil
}

${ }^{3}$ Department of Anesthesiology, Hospital de Base, Brasília, Distrito Federal, Brazil

*Corresponding author: Denismar Borges de Miranda, PhD in Tropical Medicine and Public Health from the Institute of Tropical Pathology and Public Health at the Federal University of Goiás, Physician at the Municipal Health Department of Goiânia, Goiás, Brazil

\section{ARTICLE INFO}

Received: 慧 August 10, 2020

Published: 蔧 August 24, 2020

Citation: Catia Sousa Govêia, Denismar Borges de Miranda, Larissa Govêia Moreira, Luís Cláudio Araújo Ladeira. Cardiac Arrhythmia Due to Intraorbital Hematoma During Oculoplastic Surgery Under Local Anesthesia: A Case Report. Biomed J Sci \& Tech Res 29(4)-2020. BJSTR. MS.ID.004844.

\begin{abstract}
The incidence of intraorbital hemorrhage in blepharoplasties is rare. The symptoms can be severe, such as orbital compartment syndrome and oculocardiac reflex. We describe a case of intraorbital hemorrhage manifested as sudden intraoperative arrhythmia and bradycardia during blepharoplasty surgery under local anesthesia and monitored anesthesia care. This should be considered a high-risk surgery for bleeding in ophthalmology and close hemodynamic monitoring is essential for immediate diagnosis. Also, a prompt response is of utmost importance to avoid loss of vision and to assure a positive outcome.
\end{abstract}

Keywords: Cardiac Arrhythmia; IntraorbitalHematoma; Oculoplastic Surgery; Local Anesthesia

\section{Introduction}

Blepharoplasty is considered a high-risk surgery for bleeding complications in ophthalmology, but the incidence of intraorbital hemorrhage is 55 per 100,000 [1]. Once an intraorbital hematoma occurs, the symptoms can be severe, such as orbital compartment syndrome, with consequent Retinal and visual loss, in addition to oculocardiac reflex, due to the stretching of the extraocular muscles and globe compression. Next, we describe a case of intraorbital hemorrhage associated with an oculocardiac reflex manifested as arrhythmia during blepharoplasty surgery under local anesthesia and monitored anesthesia care.

\section{Case Report}

Female patient, 62 years old, programmed to upper blepharoplasty under local infiltration anesthesia, sedation and monitored anesthesia care. Her personal medical history included an antiphospholipid syndrome and a previous stroke in cerebellum, with sequelae of convulsive episodes. At the moment of hospital admission, she was in use of valproic acid and hydroxychloroquine. Aspirin use had been interrupted seven days earlier. Physical examination was unremarkable, blood pressure: 132x85 mmHg, heart rate: $72 \mathrm{bpm}$, and normal electrocardiogram. Sedation and monitored anesthesia care began with intravenous midazolam (2.5mg), fentanyl (50 mcg) and propofol (30mg), followed by local infiltration in both upper lids with $10 \mathrm{~mL}$ of a $50 \%$ mixture of $2 \%$ lidocaine and $0.5 \%$ bupivacaine with vasoconstrictor. The procedure was initially performed on the left eye.

After the beginning of the second eye's procedure, the patient presented sinus bradycardia (heart rate: $30 \mathrm{bpm}$ ), followed by ventricular extrasystoles, pain and proptosis in left eye, leading to a diagnosis of intraorbital hematoma in the first operated eye. Immediate surgical decompression was performed, with drainage of a large amount of blood and an infusion of $20 \%$ 
mannitol was started. During drainage, she presented episodes of bradycardia and psychomotor agitation, but remained conscious and became hypertensive. At this moment, considering the known etiology of the complication and the fact that the patient remained hemodynamically stable, atropine was not administered. Midazolam (2.5mg), fentanyl (50mcg), clonidine $(75 \mathrm{mcg})$ and low dose ketamine $(10 \mathrm{mg})$ were administered, with resolution of the condition. Dexamethasone $(4 \mathrm{mg})$, tenoxicam $(40 \mathrm{mg})$ and ondansetron (4mg) were also administered. Since the operation on the second eye had already begun, the surgeon chose to continue the procedure, which resumed uneventfully. After completing the surgical plan and resolving the left intraorbital hematoma, she was referred to the post-anesthetic recovery room, where she remained monitored and without complaints for two hours. She was kept under observation in the ward and was discharged from the hospital six hours later without further complications.

\section{Discussion and Conclusions}

Intraorbital hemorrhage during blepharoplasty is an extremely rare complication and occurs due to surgical manipulation of the orbital fat without adequate hemostasis [1]. The use of aspirin is widely indicated in patients at risk for thromboembolic events and its suspension can lead to a rebound effect due to increased thromboxane A2 activity [2]. Therefore, aspirin suspension is not recommended in low-risk ophthalmic surgeries, such as cataracts, and even for medium-risk surgeries such as vitrectomy and trabeculectomy, and continuation of therapy can be maintained [3-5]. However, in the case of blepharoplasty, since it is a surgery with a higher risk of bleeding, antiplatelet therapy should be discontinued seven days before, as was done in this case. Despite the low incidence, orbital hematoma during blepharoplasty may be sufficient to produce orbital compartment syndrome, defined by increased intraocular pressure with consequent compression of the retinal artery and optic nerve. Visual loss occurs within 100 minutes if the condition is not properly treated. The most common symptoms are pain, proptosis refractory to compression, immobility of extraocular muscles, subconjunctival hemorrhage, reduced visual acuity, diplopia and increased intraocular pressure [6].

The most efficient treatment for orbital compartment syndrome is achieved through cantotomy associated with lateral cantolysis, preferably within 90 minutes after the trauma. Other measures must be performed simultaneously, such as head elevation, effective analgesia and antiemesis to improve the drainage of aqueous humor and administration of hypertonic solution or carbonic anhydrase inhibitor, in order to decrease the production of aqueous humor and, consequently, reduce intraocular pressure $[6,7]$. In the case reported, the signs presented by the patient, of pain and proptosis, suggest an increase in intraocular pressure, and treatment with mannitol and adequate hemostasis was sufficient to resolve the condition. Also, clonidine administration was applied as an adjunct to reduce intraocular pressure, as the stimulation of peripheral $\alpha$ receptors promote vasoconstrictive effects in the eye [8]. The oculocardiac reflex was first described in 1908 [9]. Triggering factors are compression of the globe or stretching of the extraocular musculature. The reflex aference is provided by stimulus of the trigeminal ophthalmic branch, and the vagal efference via muscarinic cardiac fibers leads to bradycardia and hypotension.

Other cardiac features of this reflex may manifest as arrhythmias, cardiac blocks or even asystole. There may also be an enhancement in gastric motility by intestinal parasympathetic stimulation. In the present case, the oculocardiac reflex was precipitated by the increase in intraocular pressure and stretching of the extraocular musculature due to the acute rise in intraorbital pressure. In blepharoplasties, the incidence of oculocardiac reflex is high $(40 \%)$ due to the stretching of the aponeurosis of the eyelid levator muscle [10]. This muscle is strictly related to the superior rectus muscle, so that its traction leads to the traction of the superior rectus muscle. This does not seem to have been the cause of the symptoms in the case reported, as the oculocardiac reflex tends to fatigue. Thus, it is more common to occur during surgery on the first eye, as previously published [10]. In this type of surgery, atraumatic operative management prevents the appearance of the trigeminovagal reflex. Compression of the globe should be avoided, and the lifting of the eyelid muscle aponeurosis should be slow and smooth [10].

Some authors suggest investigating patients susceptible to vagal events in the pre-anesthetic evaluation; that is, patients with a history of conduction blocks, vasovagal responses or in chronic use of beta-blockers. These patients benefit from prior administration of intravenous atropine $[9,10]$. Given the muscarinic nature of the trigeminocardiac reflex, atropine is described as first choice treatment. However, in this particular case, atropine was not immediately considered, as bradycardia did not produce signs and symptoms of low cardiac output. Instead, the patient remained conscious and even exhibited hypertension. As such, surgical decompression and other measures to lower intraocular pressure were effective in addressing the etiology and offsetting the symptoms. Even after resolution, close monitoring and follow up should be ensured, as orbital hematoma symptoms may still develop within the first 24 hours [11].

We present a case of sudden intraoperative arrhythmia and bradycardia as manifestations of an oculocardiac reflex precipitated by intraorbital hemorrhage as a complication of blepharoplasty. This should be considered a high-risk surgery for bleeding in ophthalmology and close hemodynamic monitoring is essential for immediate diagnosis. Also, a prompt response is of utmost importance to avoid loss of vision and to assure a positive outcome. 


\section{Conflict of Interest}

The authors have declared that no competing interest exists.

\section{Authors' Contributions}

CSG, LGM, and LCAL collected the patient's information drafted this manuscript and made contribution to supervision and final approval, and DBM analyzed and interpreted the patient data, and final approval. All authors read and approved the final manuscript.

\section{References}

1. Grumbine F, Deparis S, Kersten R (2015) Delayed periocular hemorrhage after upper blepharoplasty. Orbit 34: 103-105.

2. Brillat E, Rouberol F, Palombi K (2015) A case-control study to assess aspirin as a risk factor of bleeding in rhegmatogenous retinal detachment surgery. Graefes Arch Clin Exp Ophthalmol 253(3): 1899-1905.

3. Douketis JD, Spyropoulos AC, Spencer FA, Andrew S Dunn, Regina Kunz, et al. (2012) Perioperative management of antithrombotic therapy: antithrombotic therapy and prevention of thrombosis, ( $9^{\text {th }}$ Edn): american college of chest physicians' evidence-based clinical practice guidelines. Chest 141: e326S-e350S.

4. Lip GYH, Durrani OM, Roldan V (2011) Peri-operative management of ophthalmic patients taking antithrombotic therapy. Int J Clin Pract 65 361-371.

\section{ISSN: 2574-1241}

\section{DOI: 10.26717 /BJSTR.2020.29.004844}

Denismar Borges de Miranda. Biomed J Sci \& Tech Res

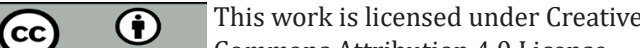

Submission Link: https://biomedres.us/submit-manuscript.php
5. Narendran N, Williamson TH (2003) The effects of aspirin and warfarin therapy on haemorrhage in vitreoretinal surgery. Acta Ophthalmol Scand 81: 38-40.

6. Romaniuk V (2013) Ocular trauma and other catastrophes. Emerg Med Clin North Am 31(12): 399-411.

7. Rowh AD, Ufberg JW, Chan TC (2015) Lateral canthotomy and cantholysis: emergency management of orbital compartment syndrome. J Emerg Med 48(3): 325-330.

8. Weigert G, Resch H, Luksch A, Leopold Schmetterer, Gerhard Garhofer, et al. (2007) Intravenous administration of clonidine reduces intraocular pressure and alters ocular blood flow. Br J Ophthalmol 91(10): 13541358.

9. Nicholson D, Kossler A, Topping K (2017) Exaggerated oculocardiac reflex elicited by local anesthetic injection of an empty orbit: a case report. A A Case Rep 9(12): 337-338.

10. Uda H, Sugawara Y, Sarukawa S (2014) The oculocardiac reflex in aponeurotic blepharoptosis surgery. J Plast Surg Hand Surg 48: 170-174.

11. Christie B, Block L, Ma Y (2018) Retrobulbar hematoma: a systematic review of factors related to outcomes. J Plast Reconstr Aesthet Surg 71(2): 155-161.

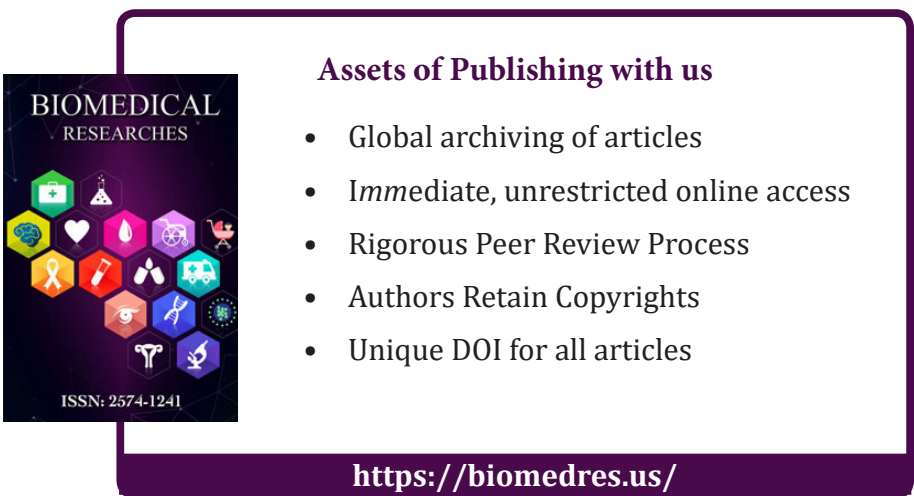

